\title{
PD-L1 and IDO-1 expression in undifferentiated pleomorphic sarcoma: The associations with tumor infiltrating lymphocytes, dMMR and HLA class I
}

\author{
SHIN ISHIHARA ${ }^{1}$, YUICHI YAMADA ${ }^{1}$, TAKESHI IWASAKI ${ }^{1}$, MASATO YOSHIMOTO ${ }^{1}$, \\ YU TODA ${ }^{1}$, KENICHI KOHASHI ${ }^{1}$, HIDETAKA YAMAMOTO ${ }^{1}$, \\ YOSHIHIRO MATSUMOTO $^{2}$, YASUHARU NAKASHIMA ${ }^{2}$ and YOSHINAO ODA $^{1}$ \\ Departments of ${ }^{1}$ Anatomic Pathology and ${ }^{2}$ Orthopedic Surgery, \\ Graduate School of Medical Sciences, Kyushu University, Fukuoka 812-8582, Japan
}

Received April 3, 2020; Accepted September 25, 2020

DOI: $10.3892 /$ or.2020.7837

\begin{abstract}
The prognosis of undifferentiated pleomorphic sarcoma (UPS) is generally unfavorable. Recently, clinical trials such as SARC028 demonstrated the utility of cancer immunotherapy for soft tissue sarcomas. The aim of the present study was to assess the expression of PD-L1 and IDO-1 as prognostic factors and therapeutic targets. A total of 52 primary UPS cases were retrieved and two UPS cell lines were utilized for supplementary analysis. Immunohistochemical staining of anti-PD-L1 (28-8), IDO-1, CD8, CD4, CD3, HLA class I, MSH2, MSH6, MLH1 and PMS2 was carried out. Immunohistochemically, 19 of 52 (36.5\%) cases showed PD-L1 expression at least focally ( $\geq 1 \%)$ and 5 of $52(9.62 \%)$ showed strong PD-L1 expression ( $\geq 50 \%)$. Overall, 25 of $52(48.1 \%)$ cases expressed IDO-1 ( $\geq 1 \%)$. Two tumors were evaluated as having deficient mismatch repair and six tumors as having the loss of HLA class I. PD-L1 expression $(\geq 1 \%)$ was significantly related to the infiltration of CD8- and CD3-positive lymphocytes, but strong PD-L1 expression $(\geq 50 \%)$ did not present a significant relationship with tumor-infiltrating lymphocytes. IDO-1 expression was also associated with CD8-, CD4-, and CD3-positive lymphocytes. In vitro, both PD-L1 and IDO-1 were induced by IFN- $\gamma$ stimulation. In survival analysis, strong PD-L1 expression $(\geq 50 \%)$ was a significant poor prognostic factor, while IDO-1 expression ( $\geq 1 \%$ ) was a favorable one. In
\end{abstract}

Correspondence to: Dr Yoshinao Oda, Department of Anatomic Pathology, Graduate School of Medical Sciences, Kyushu University, Maidashi 3-1-1, Higashi-ku, Fukuoka 812-8582, Japan

E-mail: oda@surgpath.med.kyushu-u.ac.jp

Abbreviations: UPS, undifferentiated pleomorphic sarcoma; TIL, tumor-infiltrating lymphocyte; dMMR, deficient mismatch repair

Key words: IDO-1, HLA class I, mismatch repair, PD-L1, undifferentiated pleomorphic sarcoma conclusion, UPS was shown to frequently express PD-L1 and IDO-1. It was suggested that PD-L1 expression ( $\geq 50 \%)$ and IDO-1 expression are poor and favorable prognostic factors of UPS patients, respectively.

\section{Introduction}

Undifferentiated pleomorphic sarcoma (UPS) is a soft tissue sarcoma of uncertain differentiation with prominent nuclear pleomorphism. In the current WHO classification of soft tissue tumors, UPS is defined as a subtype of undifferentiated soft-tissue sarcoma (USTS) (1). UPS has been a heterogeneous tumor category and it has usually been diagnosed by ruling out the diagnosis of other types of soft tissue sarcoma with specific differentiation. Immunohistochemically, the tumor cells of UPS present no definitive expression of specific immunohistochemical markers (1).

The prognosis of UPS remains unfavorable despite wide resection and additional chemotherapy or radiation therapy. Standard chemotherapy for UPS is anthracycline plus ifosfamide neoadjuvant chemotherapy (2). The efficacy of eribulin and the clinical outcome of pazopanib and gemcitabine/docetaxel for UPS have also been reported $(3,4)$. Cancer immunotherapy has been successfully used in some cancers such as malignant melanoma and non-small-cell lung carcinoma (5-8). SARC028, a clinical trial of anti-PD-1 therapy for patients with metastatic or surgically unresectable locally advanced sarcoma, demonstrated the effectiveness of this therapeutic approach for pleomorphic sarcomas such as UPS and dedifferentiated liposarcoma (9). Moreover, anti-PD-1 therapy was approved for the treatment of tumors with deficient mismatch repair (dMMR) (10). Deficiency of MLH1, MSH2, MSH6 and PMS2 may cause a high number of genetic mutations, especially frameshift mutations in the repetitive DNA sequences known as microsatellites, resulting in neoantigens. Thus, tumors with MSI-high may become a target of the patient's immune system. It is known that MSI-high tumors are eliminated by antitumor immunity upon treatment with immune checkpoint blockade. On the other hand, the dMMR status of UPS remains unknown (10). As HLA 
class I is required for cytotoxic immunity, the effectiveness of anti-PD-1 therapy for tumors with the loss of HLA class I, a target of cytotoxic $\mathrm{T}$ lymphocytes, is now studied and this remains controversial $(11,12)$. However, it is unclear whether UPS cases exhibit a decrease or complete loss of expression of HLA class I. Indoleamine 2,3-dioxygenase (IDO-1) is the rate-limiting enzyme in tryptophan catabolism and has a potent immune-suppressive effect through local inhibition of $\mathrm{T}$ lymphocytes and a clinical trial of therapy combining anti-PD-1 and anti-IDO-1 was also performed $(13,14)$. Although the outcome was unfavorable (15), it was recently reported that tumors expressing IDO-1 may be a good target for anti-PD-1 therapy (12). Previous findings on various cancers such as lung cancer and melanoma have also described that PD-L1 and IDO-1 are induced by TILs $(5,16,17)$. However, only a small number of studies on the expression of PD-L1 and IDO-1 in sarcoma have been carried out.

In the present study, we examined the immune microenvironment of UPS, such as the expression of immune checkpoint markers, PD-L1 and IDO-1, tumor-infiltrating lymphocytes (TILs), the status of dMMR, and the expression of HLA class I to reveal their value as prognostic factors and therapeutic targets.

\section{Materials and methods}

Patients and materials. This study was conducted in accordance with the principles embodied in the Declaration of Helsinki. The study was also approved by the Ethics Committee of Kyushu University (nos. 29-429, 29-625) and consent was obtained from the patients that donated these tissues. A total of 52 cases of UPS, previously diagnosed as malignant fibrous histiocytoma (MFH) or UPS, were retrieved from among the soft tissue tumors registered in the files of the Department of Anatomic Pathology, Graduate School of Medical Sciences, Kyushu University, Fukuoka, Japan, from 1998 January to 2017 December.

To collect pure primary UPS, tumors were assessed according to the flow chart presented in Fig. S1. Secondary sarcomas following the other distinct tumors, USTS of the spindle cell type and epithelioid USTS classified in accordance with the WHO 2013 classification were excluded (1). We also excluded sarcomas after radiation and/or chemotherapy; sarcomas located in the body cavity, retroperitoneum, or bone; sarcomas in which the proportion of myxoid matrix exceeded $10 \%$; and sarcomas that were immunoreactive for MDM2 or positive for MDM2 gene amplification in order to rule out myxofibrosarcoma (MFS) and dedifferentiated liposarcoma $(18,19)$.

Formalin-fixed, paraffin-embedded samples of the 52 tumors were available. Follow-up information was available for 49 cases. Cases without wide resection were removed. Finally, 42 cases were analyzed for overall, metastasis-free, and recurrence-free survival. The follow-up period after surgery ranged from 7 to 140 months (38.5 months as the average).

Clinicopathological and histological evaluation. Clinical and pathological data were obtained from the database of the Department of Anatomic Pathology, Kyushu University. Clinicopathological findings such as age, sex, tumor location, tumor size, distant metastasis, and local recurrence were evaluated. The cases were classified into younger and older groups according to the median age. The cases were also classified into smaller and larger groups according to the cut-off of $5 \mathrm{~cm}$. We also evaluated histopathological findings such as necrosis, mitosis, and grade of the French Federation of Cancer Centers (FNCLCC) grading system of the primary tumors (20). The existence of necrosis was classified as 'positive' for necrosis. Mitosis was classified as 'high; when there were $>10$ mitotic figures per 10 high power fields (HPFs). The existence of a myxoid area (1-10\%) of the total area was classified as 'positive' for focal myxoid area.

Immunohistochemistry (IHC). Formalin fixation was carried out with $10 \%$ formalin at about $18^{\circ} \mathrm{C}$ for $48-72 \mathrm{~h}$ following tissue resection. Formalin-fixed, paraffin-embedded (FFPE) samples of 52 tumors were available for IHC staining of PD-L1 (28-8), IDO-1, CD8, CD4, CD3, and HLA class I. Such samples of 50 tumors were available for immunohistochemical staining of MSH2, MSH6, MLH1, and PMS2. FFPE samples of two cases were used up and not available for immunostaining of dMMR. FFPE tissue was sectioned at a thickness of $3 \mu \mathrm{m}$. Primary antibodies for the immunohistochemical staining of PD-L1 (28-8), IDO-1, CD8, CD4, CD3, HLA class I, MLH1, PMS2, MSH2, and MSH6 were used as described in Table SI. The immunoperoxidase polymer method (Envision-kit and Envision Flex-kit; Dako Japan) was performed for all available cases. Antigen retrieval was carried out by boiling the slides with $10 \mathrm{mM}$ sodium citrate ( $\mathrm{pH}$ 6.0) or Target Retrieval Solution (pH 9.0; Dako, Carpinteria).

The expression of PD-L1 was assessed by determining the ratio of membranous staining-positive tumor cells to all tumor cells in a stained slide in which at least 100 tumor cells were observed, in accordance with a previously reported standardized method (21), regardless of the staining intensity. We set the cut-off of PD-L1 expression as 1 or $50 \%$ in accordance with the trial $(22,23)$. The expression of IDO-1 was assessed by determining the ratio of cytoplasmic staining-positive tumor cells to all tumor cells in a stained slide in which at least 100 tumor cells were observed. We also set the cut-off of IDO-1 expression as $1 \%$, with reference to the literature $(21,24)$. Macrophages were also stained by the immunohistochemical staining of PD-L1 and IDO-1, but they were excluded from the count of positive-stained cells.

TILs were assessed by counting the number of CD8-, CD4-, and CD3-positive lymphocytes infiltrating into the tumor cells per five high-power fields (HPFs), which were randomly chosen in tumors excluding the fields with lymphoid aggregates (25). For survival analysis, the number of infiltrated lymphocytes was divided into high and low groups according to the cut-off determined by drawing ROC curves.

The expression of MSH2, MSH6, MLH1 and PMS2 was classified as having been lost when there was a complete absence of nuclear staining in neoplastic cells, while the surrounding non-neoplastic cells showed consistently preserved nuclear staining (26). Thus, antibodies for HLA class I-A, B and C were used. The expression of HLA class I was classified in accordance with a previous study (27). When the cell membrane was stained as strongly as stromal lymphocytes or endothelial cells in $>75 \%$ of the tumor cells, expression levels were defined as strong. If heterogeneous membranous 
staining was found in $>25 \%$ of the tumor cells, expression was defined as weak. If $<75 \%$ of the tumor cells lacked membrane staining, it was defined as no expression. Cases with weak and no expression were classified as those with loss of HLA class I and cases with strong expression as having HLA class I intact. To confirm that CD8-, CD4-, and CD3-positive lymphocytes secreted IFN- $\gamma$, the EnVision ${ }^{\mathrm{TM}}$ Gl2 Doublestain System was used to stain IFN- $\gamma$ and CD8, CD4, or CD3 at the same time in 10 samples each, in accordance with the basic method. IFN- $\gamma$ was colored red using alkaline phosphatase and CD8, CD4, and CD3 were colored brown using peroxidase.

Cell culture and cytokine experiments. UPS cell lines, FPS-1 and FU-MFH2, were cultured in RPMI-1640 medium and Dulbecco's modified Eagle's medium (DMEM)/F-12 supplemented with $10 \%$ fetal bovine serum and $1 \%$ penicillin/streptomycin $(28,29)$. Mycoplasma testing had been done. These cells were cultured in six-well plates in the presence or absence of IFN- $\gamma(200 \mathrm{ng} / \mathrm{ml})$ for $24 \mathrm{~h}$ in $37^{\circ} \mathrm{C}$. Then, mRNA and protein were extracted as described below.

RNA isolation, reverse transcription, and qPCR of cell lines. Cultured cells were suspended in QIAzol solution (Qiagen). Total RNA of cultured cells was extracted with acidic phenol: Chloroform and then reverse-transcribed using ReverTra Ace qPCR RT Master Mix with gDNA Remover (Toyobo), in line with the manufacturer's recommendations. Quantitative polymerase chain reaction (qPCR) for PD-L1 and IDO-1 was performed using THUNDERBIRD SYBR qPCR Master Mix (Toyobo) on an Applied Biosystems Step One Plus Real Time PCR System (Thermo Fisher Scientific Inc.). Primers of PD-L1 and IDO-1 are listed in Table SII. The data were normalized to GAPDH expression levels and are presented as the mean \pm standard deviation of three independent experiments.

Immunoblotting. Cells were washed twice with phosphate-buffered saline (PBS) and suspended in $2 \mathrm{X}$ sodium dodecyl sulfate (SDS) sample buffer of $37^{\circ} \mathrm{C}$. The samples were separated by SDS-PAGE (5-20\% gel) and transferred to a polyvinylidene fluoride membrane with the Trans-Blot Turbo Transfer System (Bio-Rad Laboratories; 2.5 A, 25 V, 7 min). Membranes were blocked for $30 \mathrm{~min}$ in 5\% Blocking One-P (Nacalai Tesque Inc.) and then incubated with primary antibodies in Can Get Signal (Toyobo). The primary antibodies used included rabbit monoclonal anti-PD-L1 (E1L3N, 1:1,000; Cell Signaling Technology), mouse anti-IDO-1 (UMAB126, 1:1,000; OriGene), and rabbit anti-HSP 90 (C45G5, 1:1,000; Cell Signaling Technology). The membranes were then washed with TBS containing $0.05 \%$ Tween-20 and incubated with horseradish peroxidase (HRP)-conjugated anti-rabbit IgG (7074S, 1:1,000; Cell Signaling Technology) or anti-mouse IgG (sc-2005, 1:1,000; Santa Cruz). The membranes were then washed and developed to enhance the chemiluminescence with Chemi-Lumi One Ultra (Nacalai Tesque Inc.). The antibodies are described in Table SI. The chemiluminescence signals were detected with an Image Quant LAS 4000 (Fujifilm). ImageJ was used to analyze the data.

Statistical analysis. Cut-offs of PD-L1 expression of 1 and $50 \%$ were set and statistical analyses between $<1 \%$ and $\geq 1 \%$ as well as $<50 \%$ and $\geq 50 \%$ were performed $(22,23)$. The cut-off of IDO-1 expression of $1 \%$ was set and the two groups were analyzed accordingly $(21,24)$. Clinicopathological and immunohistochemical parameters were analyzed using the Fisher's exact test. The infiltration of lymphocytes was analyzed by Mann-Whitney $U$ test and linear regression analysis. Correlation between the immune checkpoints and TILs was assessed by applying the least squares method. Survival curves were created using the Kaplan-Meier method. Overall, metastasis-free, and recurrence-free survival curves were analyzed by the log-rank test. For survival analysis and the Fisher's exact test, the cut-offs of TILs were determined by drawing ROC curves. The outcome of qPCR was analyzed by the Student's t-test. A P-value of $<0.05$ was classified as significant for each statistical analysis. Statistical analyses were conducted using the JMP statistical software package (version 13; SAS Institute).

\section{Results}

Clinicopathological and histological findings. The clinicopathological data for the 52 tumors are summarized in Table I. The patients included 26 males and 26 females, with ages ranging from 38 to 90 years (median: 69.5, mean: 69.1 years) at the diagnosis of the primary lesion. Thirty-two tumors $(72.7 \%)$ were $>5 \mathrm{~cm}$. Three cases $(5.8 \%)$ were located in the head and neck ( 1 case in the head and 3 in the neck), 8 cases (15.4\%) in the trunk ( 2 cases in the abdominal wall and 6 in the back), 9 cases $(17.3 \%)$ in an upper extremity (3 cases in the upper arm and 6 in the forearm), and 32 cases (61.6\%) in a lower extremity ( 25 cases in the thigh and 7 in the lower leg). Tumor size ranged from 2.5 to $20 \mathrm{~cm}$ (median: $7.5 \mathrm{~cm}$, mean: $8.3 \mathrm{~cm}$ ). Tumor-related death occurred in 13 of 42 cases (31.0\%), distant metastasis in 18 of 42 cases $(42.9 \%)$, and local recurrence in 11 of 42 cases $(26.2 \%)$. Metastatic sites were as follows: Lung in 11 cases $(61.1 \%)$, bone in 3 cases $(16.7 \%)$, lymph node in 2 cases $(11.1 \%)$, and abdominal wall in 1 case $(5.6 \%)$, as described in Table SIII.

A representative case of each histological feature is shown in Fig. 1. All 52 tumors showed the proliferation of spindle- to polygonal-shaped tumor cells with high-grade nuclear atypia (Fig. 1A), accompanied by no or only a focal $(\leq 10 \%)$ myxoid area. Atypical tumor giant cells were scattered (Fig. 1B). Tumor necrosis (Fig. 1C) was evidenced in 32 of 52 cases $(61.5 \%)$ and mitosis ( $\geq 10 / 10 \mathrm{HPFs}$ ) (Fig. 1D) was observed in 29 of $52(55.8 \%)$. Overall, 26 of 52 cases were classified as FNCLCC grade $2(50.0 \%)$ and 26 as grade $3(50.0 \%)$. Focal myxoid area $(\geq 1$ and $<10 \%)$ was observed in 21 of 52 cases (40.4\%) (Fig. 1E).

Immunohistochemistry (PD-L1, IDO-1, TILs, HLA class I, $d M M R)$. The results of the immunohistochemical study, the positive ratio of PD-L1 and IDO-1 and whether there were UPS with dMMR of loss of HLA class I are summarized in Table II. Representative figures of immunohistochemical staining are shown in Fig. 2. Regarding the immunohistochemical results of PD-L1 immunostaining, 33 of $52(63.5 \%)$ cases showed negative staining ( $<1 \%$ : Fig. $2 \mathrm{~A}), 14$ of $52(26.9 \%)$ showed focal staining ( $\geq 1$ and $<50 \%$ : Fig. $2 \mathrm{~B}$ ), and 5 of 52 $(9.6 \%)$ showed strong staining ( $\geq 50 \%$; Fig. $2 \mathrm{C})$. Regarding 
Table I. Clinicopathological and histological features.

\begin{tabular}{|c|c|}
\hline Characteristics & No. $(\%)$ \\
\hline \multicolumn{2}{|l|}{ Age $($ Average $=69.5)$} \\
\hline$(<70)$ & $26 / 52(50.0)$ \\
\hline$(\geq 70)$ & $26 / 52(50.0)$ \\
\hline \multicolumn{2}{|l|}{ Sex } \\
\hline Female & $24 / 52(46.2)$ \\
\hline Male & $28 / 52(53.8)$ \\
\hline \multicolumn{2}{|l|}{ Size } \\
\hline Smaller group $(\leq 5 \mathrm{~cm})$ & $12 / 44(27.3)$ \\
\hline Larger group $(>5 \mathrm{~cm})$ & $32 / 44(72.7)$ \\
\hline \multicolumn{2}{|l|}{ Localization } \\
\hline Head and neck & $3 / 52(5.8)$ \\
\hline Anterior trunk & $2 / 52(3.9)$ \\
\hline Back & $6 / 52(11.5)$ \\
\hline Upper arm & $3 / 52(5.8)$ \\
\hline Forearm & $6 / 52(11.5)$ \\
\hline Thigh & $25 / 52(48.1)$ \\
\hline Lower leg & $7 / 52(13.5)$ \\
\hline \multicolumn{2}{|l|}{ Metastasis } \\
\hline- & $24 / 42(57.1)$ \\
\hline+ & $18 / 42(42.9)$ \\
\hline \multicolumn{2}{|l|}{ Recurrence } \\
\hline- & $31 / 42(73.8)$ \\
\hline+ & $11 / 42(26.2)$ \\
\hline \multicolumn{2}{|l|}{ Necrosis } \\
\hline- & $32 / 52(61.5)$ \\
\hline+ & $20 / 52(38.5)$ \\
\hline \multicolumn{2}{|l|}{ Mitosis } \\
\hline Low (<10/10HPFs) & $23 / 52(44.2)$ \\
\hline High ( $\geq 10 / 10 H P F s)$ & $29 / 52(55.8)$ \\
\hline \multicolumn{2}{|l|}{ FNCLCC } \\
\hline Grade 2 & $26 / 52(50.0)$ \\
\hline Grade 3 & $26 / 52(50.0)$ \\
\hline \multicolumn{2}{|l|}{ Myxoid area } \\
\hline$<1 \%$ & $31 / 52(59.6)$ \\
\hline $1-10 \%$ & $21 / 52(40.4)$ \\
\hline
\end{tabular}

IDO-1, 27 of $52(51.9 \%)$ cases showed negative staining $(<1 \%$ : Fig. 2D), 17 of 52 (32.7\%) showed focal staining ( $\geq 1$ and $<10 \%$ : Fig. 2E), 8 of $52(15.4 \%)$ showed moderate staining ( $\geq 10$ and $<25 \%$ : Fig. $2 \mathrm{~F}$ ), and none showed strong staining ( $\geq 25 \%)$. The numbers of CD8-positive lymphocytes (Fig. 2G) ranged from 0 to 489 per five HPFs, with a median of 62.5 . The numbers of CD4-positive lymphocytes (Fig. $2 \mathrm{H}$ ) ranged from 1 to 221 per five HPFs, with a median of 22.5. The numbers of CD3-positive lymphocytes (Fig. 2I) ranged from 1 to 811 per five HPFs, with a median of 117. There was deficiency of mismatch repair protein in 2 of 50 tumors $(4.0 \%)$ : One case with loss of MSH2 and MSH6 (2.0\%), and another with loss of PMS2 (2.0\%) (Fig. 2J-L). For HLA class I, two tumors were classified to have no expression, four to have weak expression,
Table II. Immunohistochemical results.

\begin{tabular}{lcc}
\hline Antibody & Positive ratio & No. $(\%)$ \\
\hline PD-L1 (28-8) & $<1 \%$ & $33 / 52(63.5)$ \\
& $1 \% \leq$ and $<50 \%$ & $14 / 52(26.9)$ \\
& $\geq 50 \%$ & $5 / 52(9.6)$ \\
IDO-1 & $<1 \%$ & $27 / 52(51.9)$ \\
& $1 \% \leq$ and $<10 \%$ & $17 / 52(32.7)$ \\
& $10 \% \leq$ and $<25 \%$ & $8 / 52(15.4)$ \\
MMR & $\geq 25 \%$ & $0 / 52$ \\
& Deficient & $2 / 50(4.0)$ \\
HLA Class I & Proficient & $48 / 50(96.0)$ \\
& Loss & $6 / 52(11.5)$ \\
& Retain & $46 / 52(88.5)$ \\
\hline
\end{tabular}

and the other 46 to have strong expression (Fig. 2M and N). Therefore, six tumors were classified as having loss of HLA class I. The remaining cases were classified as having normal HLA class I and their HLA class I expression was completely retained. Double staining of IFN- $\gamma$ and CD8, CD4, or CD3 is shown in Fig. S2. CD8-, CD4-, or CD3-positive TILs infiltrating into the tumors seemed to secrete IFN- $\gamma$.

Correlations between clinicopathological features and the expression of PD-LI and IDO-1, TILs, loss of HLA class I, and $A M M R$. The results of statistical analysis between the clinicopathological features and the expression of PD-L1 or IDO-1 are summarized in Table III. ROC curves of TILs are presented in Fig. S3. Focal PD-L1 expression $(\geq 1 \%)$ was associated with necrosis $(\mathrm{P}=0.0402)$. No significant correlations between the clinicopathological features and TILs, loss of HLA class I, and dMMR were identified.

Correlations between the expression of PD-LI or IDO-1, loss of HLA class I, and dMMR. No association between the expression of PD-L1 and IDO-1 was detected by Fisher's exact test, as shown in Table SIV. Of the six tumors with loss of HLA class I, one tumor exhibited the loss of MSH2 and MSH6 and expressed focal PD-L1 but did not express IDO-1, three expressed IDO-1 but did not express PD-L1, and two expressed neither PD-L1 nor IDO-1. In the two tumors with dMMR, one tumor with the loss of MSH2 and MSH6 expressed focal PD-L1 but did not express IDO-1. The other one with the loss of PMS2 expressed strong PD-L1 and focal IDO-1.

Correlations between TILs and the expression of PD-L1 or IDO-1. The relationships between PD-L1 or IDO-1 immunoexpression and lymphocytic infiltration are presented in Fig. 3. In the Mann-Whitney U-test, PD-L1 expression ( $\geq 1 \%$ ) was associated with $\mathrm{CD} 8-(\mathrm{P}=0.0055$, Fig. $3 \mathrm{~A})$ and $\mathrm{CD} 3$-positive lymphocytes $(\mathrm{P}<0.0001$, Fig. 3C), but not with $\mathrm{CD} 4-$ positive ones (Fig. 3B). Strong PD-L1 expression ( $\geq 50 \%$ ) was not associated with lymphocytic infiltration (Fig. 3D-F). IDO-1 expression $(\geq 1 \%)$ was related to $C D 8-(P<0.0001$, Fig. $3 G)$, CD4- ( $\mathrm{P}<0.0499$, Fig. 3H), and CD3-positive lymphocytes $(\mathrm{P}=0.0003$, Fig. 3I). From the analysis applying the least 

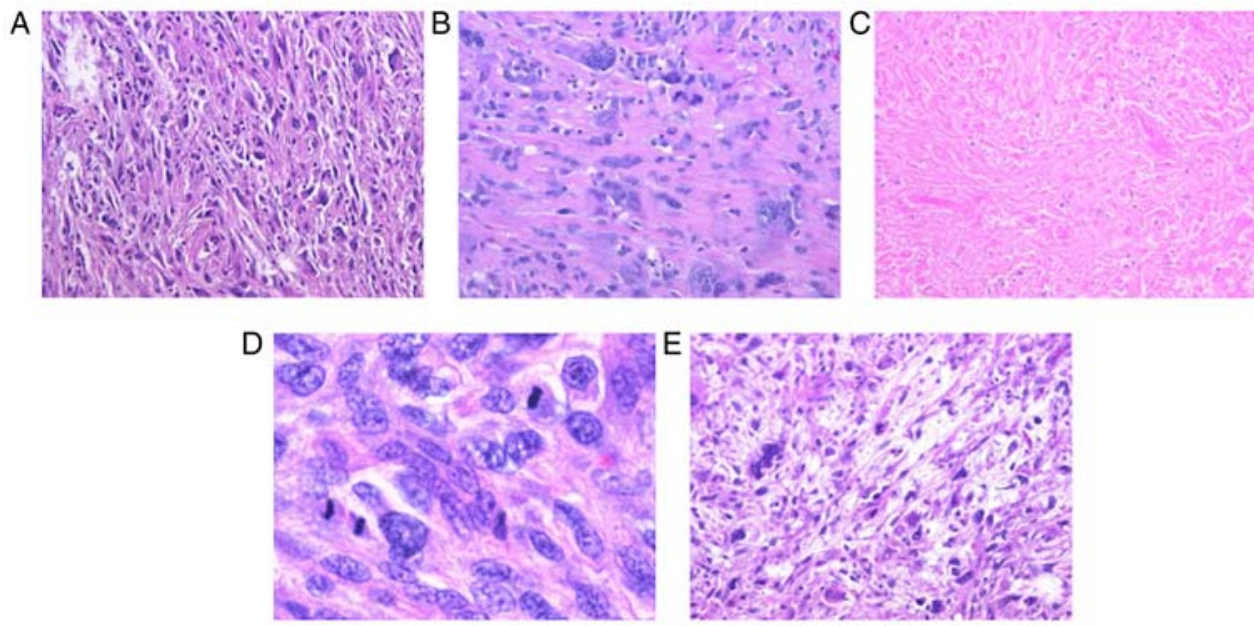

Figure 1. Histological features from hematoxylin and eosin staining in UPS. (A) Pleomorphic nuclei arranged haphazardly. (B) Atypical giant cells in UPS (C) Necrotic area in UPS. (D) Mitosis in UPS. (E) Myxoid area in UPS

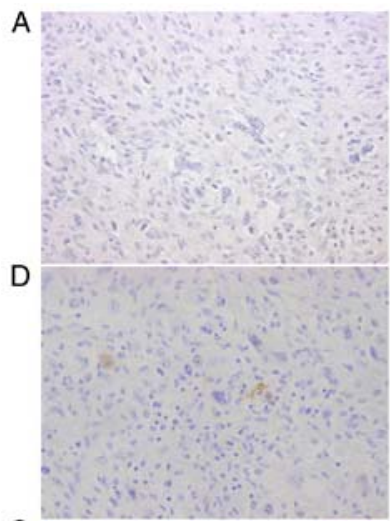

G
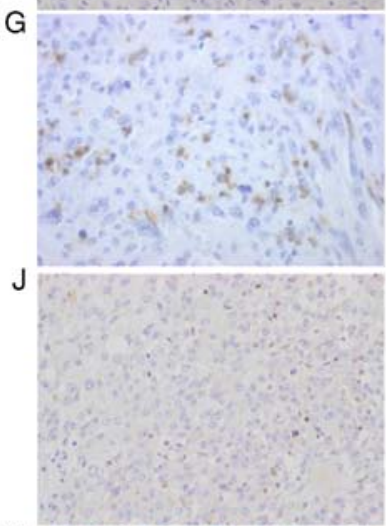

$\mathrm{M}$

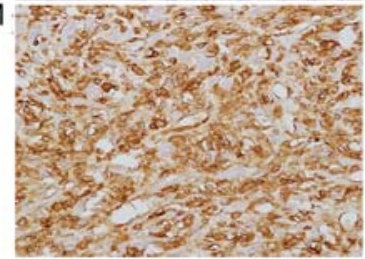

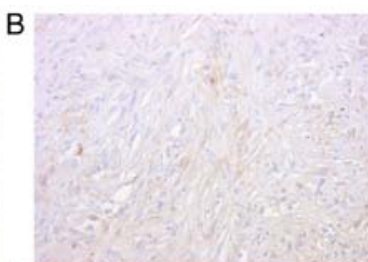
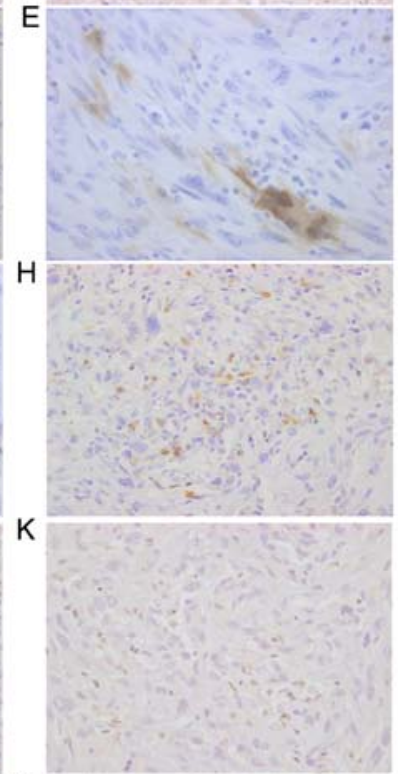

$\mathrm{N}$

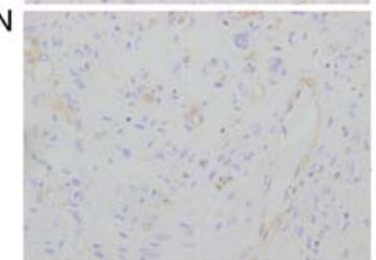

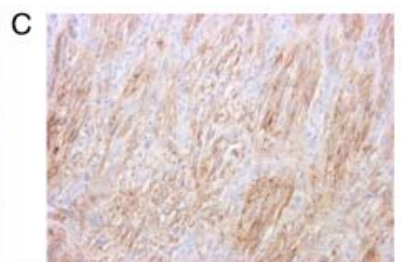

$\mathrm{F}$
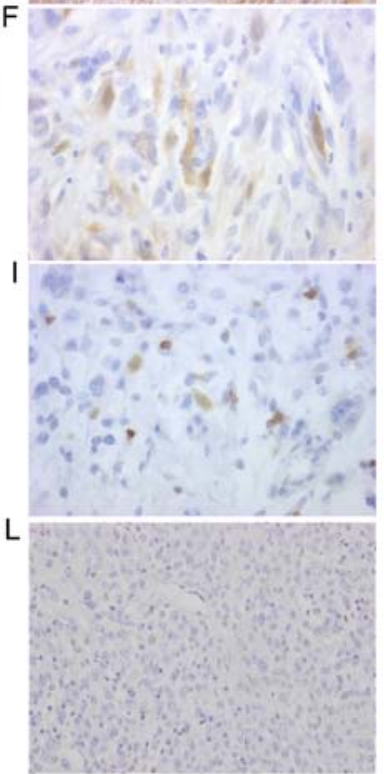

Figure 2. Immunoreactive features in UPS. (A) PD-L1: $<1 \%$. (B) PD-L1: $\geq 1 \%$ and $<50 \%$. (C) PD-L1: $\geq 50 \%$. (D) IDO-1: $<1 \%$. Macrophages were stained (E) IDO-1: $\geq 1 \%$ and $<10 \%$. (F) IDO-1: $\geq 10 \%$ and $<25 \%$. (G) Tumor-infiltrating CD8-positive lymphocytes. (H) Tumor-infiltrating CD4-positive lymphocytes. (I) Tumor-infiltrating CD3-positive lymphocytes. (J) Loss of MSH2. (K) Loss of MSH6. (L) Loss of PMS2. (M) Normal expression of HLA class I. (N) Loss of HLA class I.

squares method, the regressions between PD-L1 and CD8-, CD4-, and CD3-positive TILs were not significant (R-squared: 0.000341, 0.00158, 0.0270, P-values: 0.8966, 0.7797, 0.2440;
Fig. 3J-L). Positive correlations of IDO-1 with CD8 and CD3 were seen (R-squared: 0.270, 0.387, P-values: $<0.0001$, $<0.0001$; Fig. 3M and O). The regression between IDO-1 and 
Table III. Statistical results of clinicohistopathological and mmunohistochemical features.

\begin{tabular}{|c|c|c|c|c|c|c|}
\hline & \multicolumn{2}{|c|}{ PD-L1 (1\% cut-off) } & \multicolumn{2}{|c|}{ PD-L1 (50\% cut-off) } & \multicolumn{2}{|c|}{ IDO- 1 ( $1 \%$ cut-off) } \\
\hline & $<1 \%$ & $\geq 1 \%$ & $<50 \%$ & $\geq 50 \%$ & $<1 \%$ & $\geq 1 \%$ \\
\hline \multicolumn{7}{|l|}{ Age } \\
\hline$<70$ & 20 & 6 & 25 & 1 & 16 & 10 \\
\hline \multirow[t]{2}{*}{$\geq 70$} & 13 & 13 & 22 & 4 & 11 & 15 \\
\hline & \multicolumn{2}{|c|}{$\mathrm{P}=0.0828$} & \multicolumn{2}{|c|}{$\mathrm{P}=0.350$} & \multicolumn{2}{|c|}{$\mathrm{P}=0.267$} \\
\hline \multicolumn{7}{|l|}{ Sex } \\
\hline $\mathrm{F}$ & 16 & 8 & 22 & 2 & 11 & 13 \\
\hline \multirow[t]{2}{*}{ M } & 17 & 11 & 25 & 3 & 16 & 12 \\
\hline & \multicolumn{2}{|c|}{$\mathrm{P}=0.775$} & \multicolumn{2}{|c|}{$\mathrm{P}=1.000$} & \multicolumn{2}{|c|}{$\mathrm{P}=0.578$} \\
\hline \multicolumn{7}{|l|}{ Size } \\
\hline Small $(\leq 5 \mathrm{~cm})$ & 6 & 6 & 11 & 1 & 6 & 6 \\
\hline \multirow[t]{2}{*}{ Large $(>5 \mathrm{~cm})$} & 21 & 11 & 28 & 4 & 17 & 5 \\
\hline & \multicolumn{2}{|c|}{$\mathrm{P}=0.4889$} & \multicolumn{2}{|c|}{$\mathrm{P}=1.000$} & \multicolumn{2}{|c|}{$\mathrm{P}=1.000$} \\
\hline \multicolumn{7}{|l|}{ FNCLCC } \\
\hline Grade 2 & 20 & 6 & 26 & 0 & 12 & 14 \\
\hline \multirow[t]{2}{*}{ Grade 3} & 13 & 13 & 21 & 5 & 15 & 11 \\
\hline & \multicolumn{2}{|c|}{$\mathrm{P}=0.083$} & \multicolumn{2}{|c|}{$\mathrm{P}=0.0506$} & \multicolumn{2}{|c|}{$\mathrm{P}=0.578$} \\
\hline \multicolumn{7}{|l|}{ Necrosis } \\
\hline- & 24 & 8 & 30 & 2 & 15 & 17 \\
\hline \multirow[t]{2}{*}{+} & 9 & 11 & 17 & 3 & 12 & 8 \\
\hline & \multicolumn{2}{|c|}{$\mathrm{P}=0.0402^{\mathrm{a}}$} & \multicolumn{2}{|c|}{$\mathrm{P}=0.3607$} & \multicolumn{2}{|c|}{$\mathrm{P}=0.404$} \\
\hline \multicolumn{7}{|l|}{ Mitosis } \\
\hline Low $(<10 / 10 \mathrm{HPFs})$ & 18 & 5 & 23 & 0 & 13 & 10 \\
\hline \multirow[t]{2}{*}{ High $(\geq 10 / 10 \mathrm{HPFs})$} & 15 & 14 & 24 & 5 & 14 & 15 \\
\hline & \multicolumn{2}{|c|}{$\mathrm{P}=0.0811$} & & & & \\
\hline Myxoid area & & & & & & \\
\hline 19 & 12 & 26 & 5 & 17 & 14 & \\
\hline$<1 \%$ & 14 & 7 & 21 & 0 & 10 & 11 \\
\hline$\leq 1 \%,<10 \%$ & & & & & & \\
\hline
\end{tabular}

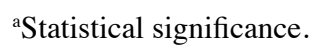

CD4 was not significant (R-squared: 0.0373, P-value: 0.1699; Fig. 3N).

Induction of PD-L1 and IDO-1 expression by IFN- $\gamma$ in vitro. The data acquired from qPCR are presented in Fig. 4A and B. IFN- $\gamma$ induced the mRNA expression of PD-L1 $(\mathrm{P}=0.0136$ and $\mathrm{P}=0.0015$, Fig. 4A) and IDO- $1(\mathrm{P}=0.0041$ and $\mathrm{P}=0.0031$, Fig. 4B). The results of western blotting showed that the expression of PD-L1 induced by IFN- $\gamma$ was 4.29 -fold as high as in the control in FPS-1 cells and 2.00-fold in FU-MFH2 cells. The expression of IDO-1 induced by IFN- $\gamma$ was higher than the expressions in the control in FPS-1 cells and in FU-MFH2 cells (Fig. 4C). IDO-1 expression of the cell lines tended to react more strongly to IFN- $\gamma$ stimulation than that of PD-L1 in both qPCR and western blotting.

Survival analysis. The results of survival curve analysis are presented in Fig. 5. Overall survival rate is shown (Fig. 5A).
Metastasis was closely related to a poorer overall survival rate $(\mathrm{P}<0.0001$, Fig. 5B), but recurrence was not in this study. Patients with strong PD-L1 expression $(\geq 50 \%)$ had worse prognosis than the other patients $(<50 \%)(\mathrm{P}=0.0229$, Fig. $5 \mathrm{C})$. On the other hand, IDO-1 expression $(\geq 1 \%)$ was associated with a better prognosis than lack of this IDO-1 expression regarding overall survival ( $\mathrm{P}=0.0056$, Fig. 5D). PD-L1 expression ( $\geq 1 \%$ ), TILs, loss of HLA class I and dMMR were not significant prognostic factors for overall survival. In addition, patients with strong PD-L1 expression also had worse prognosis than the other patients $(\mathrm{P}=0.0116$ Fig. 5E). On the other hand, IDO-1 expression ( $\geq 1 \%)$ tended to be a factor associated with a favorable prognosis compared with negative IDO-1 expression ( $\mathrm{P}=0.0630$, Fig. 5F), but it was not significant. TILs, loss of HLA class I, and dMMR were not significant prognostic factors for metastasis-free survival. No significant findings for recurrence-free survival were made for the expression of PD-L1 and IDO-1, clinicopathological features, TILs, loss of HLA class I, and dMMR. 

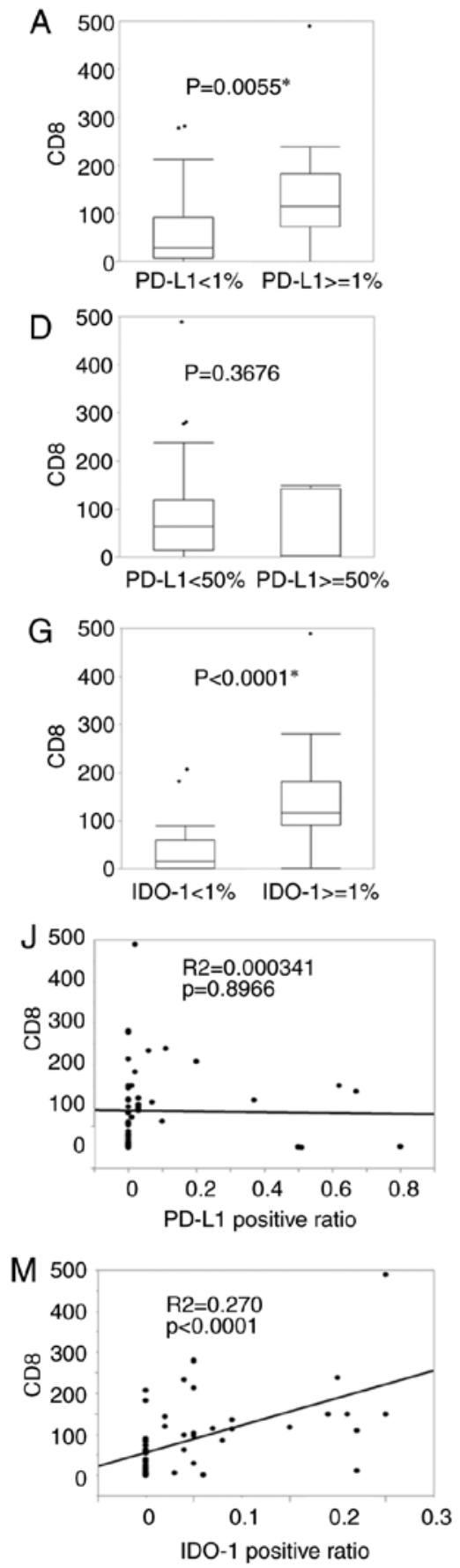
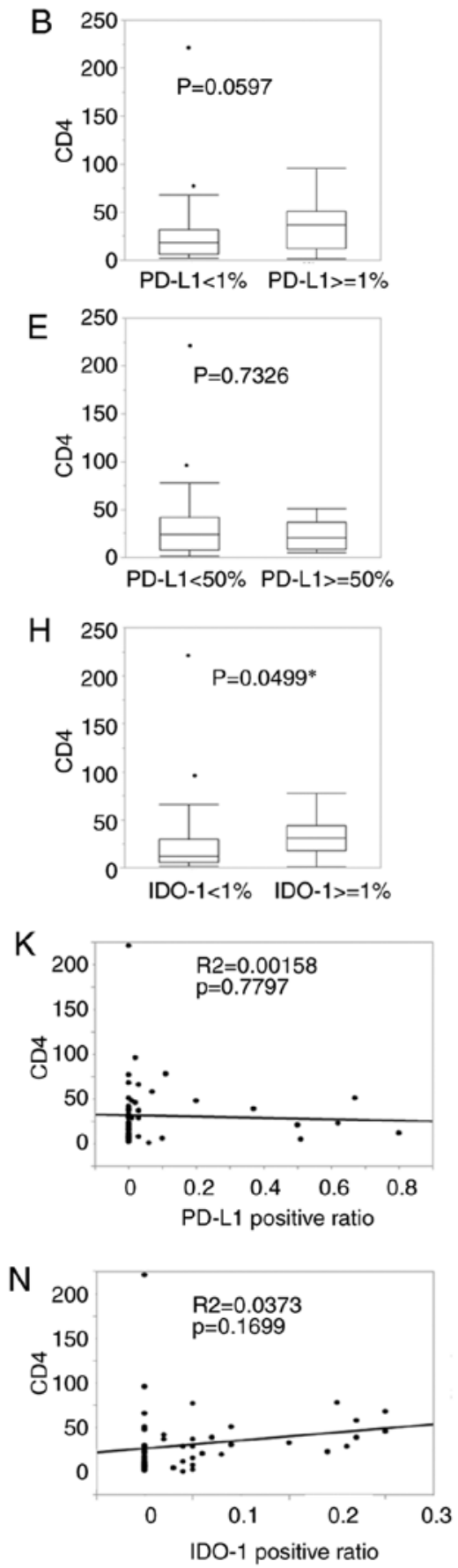
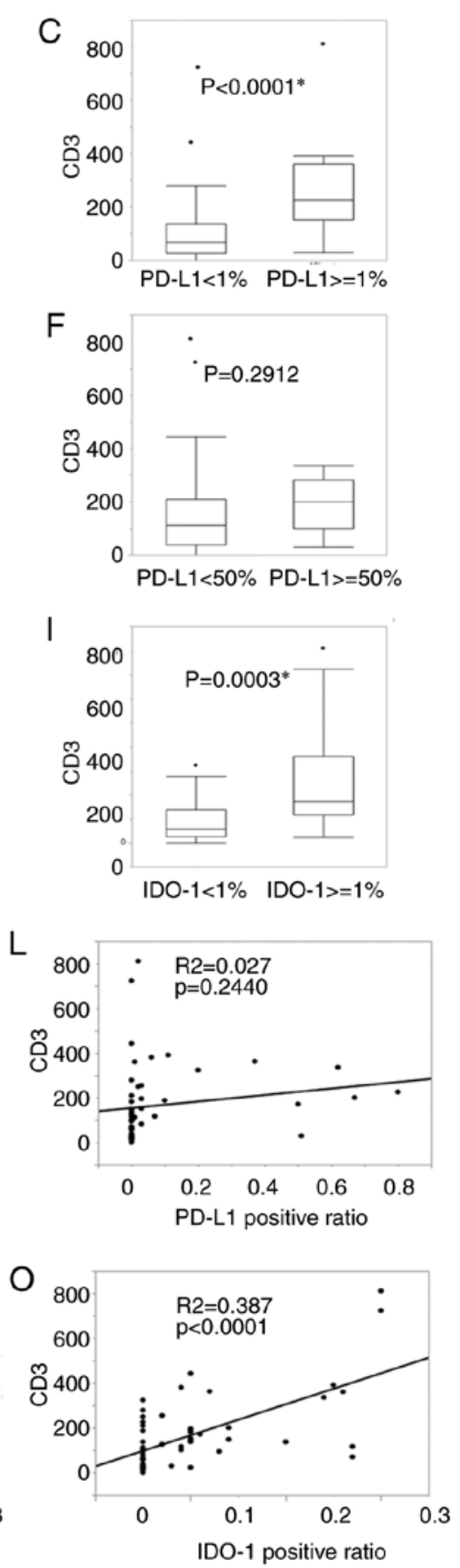

Figure 3. (A) The association between focal PD-L1 expression and the number of infiltrated CD8-positive lymphocytes (P=0.0055). (B) The association between focal PD-L1 expression and the number of infiltrated CD4-positive lymphocytes ( $\mathrm{P}=0.0597)$. (C) The association between focal PD-L1 expression and CD3 (P<0.0001). (D) The association between strong PD-L1 expression and CD8 (P=0.3676). (E) The association between strong PD-L1 expression and CD4 ( $\mathrm{P}=0.7326)$. ( $\mathrm{F})$ The association between strong PD-L1 expression and CD3 $(\mathrm{P}=0.2912)$. (G) The association between focal IDO-1 expression and CD8 $(\mathrm{P}<0.0001)$. $(\mathrm{H})$ The association between focal IDO-1 expression and CD4 ( $\mathrm{P}=0.0499)$. (I) The association between focal IDO-1 expression and CD3 $(\mathrm{P}=0.0003)$. Strong PD-L1 expression was not related to lymphocytic infiltration entirely. (J-L) PD-L1 was not significantly correlated with CD8, CD4, and CD3. (M-O) IDO-1 was significantly correlated with CD8 and CD3, but not with CD4.

\section{Discussion}

We confirmed the frequent expression of PD-L1 and IDO-1 in UPS by immunohistochemical analysis. The proportion of cases with PD-L1 expression was similar to that in a study of UPS performed by Boxberg et al (30). In detail, over 30\% of UPS cases exhibited at least focal PD-L1 expression and nearly $10 \%$ had strong PD-L1 expression.
It has been reported that PD-L1 and IDO-1 expression is associated with TILs. In the current study, PD-L1 expression $(\geq 1 \%)$ was related to the infiltration of CD8- and CD3-positive lymphocytes. IDO-1 expression $(\geq 1 \%)$ was associated with the infiltration of CD8-, CD4-, and CD3-positive lymphocytes. Of note, the group with a strong expression of PD-L1 $(\geq 50 \%)$ was not significantly associated with TILs. In the regression analysis, the correlation between PD-L1 and 
A

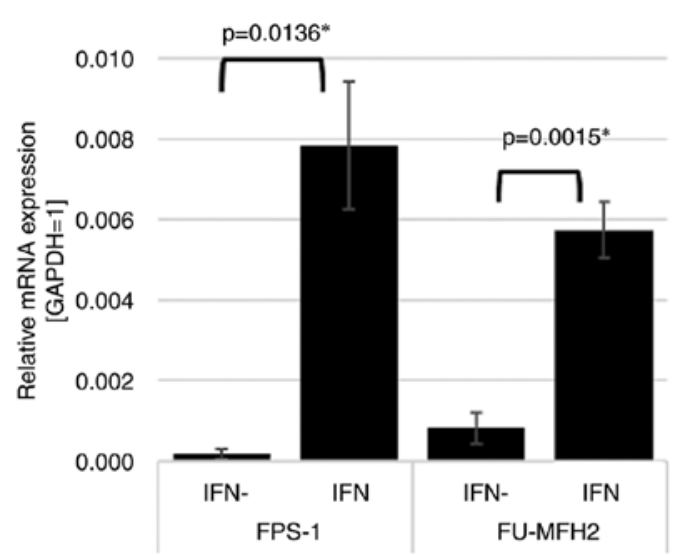

B

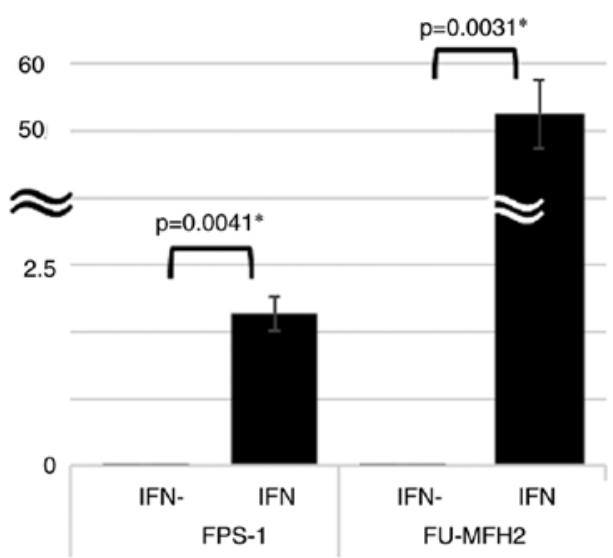

C

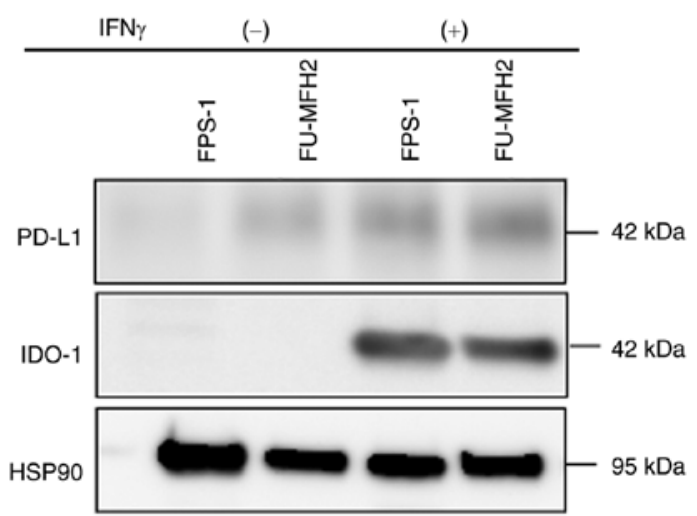

Figure 4. (A) UPS cell lines, FPS-1 and FU-MFH2, had higher PD-L1 expression when stimulated by IFN- $\gamma$ than when left unstimulated. (B) They also expressed IDO-1 much more strongly when stimulated by IFN- $\gamma$ than when left unstimulated. Upon stimulation with IFN- $\gamma$, the amount of mRNA became 300 -fold (FPS-1) and 16,000-fold (FU-MFH2) as high as that without such stimulation. (C) In western blotting, the expression of PD-L1 induced by IFN- $\gamma$ was 4.29-fold as high as that in the control in FPS-1 cells and 2.00-fold in FU-MFH2 cells. The expression of IDO-1 induced by IFN- $\gamma$ was higher than that in the control in FPS-1 cells and in FU-MFH2 cells. In this experiment, IDO-1 expression of cell lines reacted much more strongly to IFN- $\gamma$ stimulation than that of PD-L1 in both qPCR and western blotting.

TILs was not significant, but there were significant positive correlations of IDO-1 with CD8 and CD3. It was previously reported that helper $\mathrm{T}$, cytotoxic $\mathrm{T}$, and $\mathrm{NK}$ cells, as well as macrophages, secrete IFN- $\gamma(17)$ and that IFN- $\gamma$ induces the expression of PD-L1 and IDO-1 $(5,16,17)$. In the current study, PD-L1 and IDO-1 expression in UPS-cell lines were induced by IFN- $\gamma$ in vitro. The results of the current study confirmed that PD-L1 and IDO-1 were induced by TILs in sarcoma as in other cancers (5). The results also suggest that a strong PD-L1 expression $(\geq 50 \%)$ requires factors other than TILs. These other factors may be intrinsic to cancer, such as copy number gain and CMTM6 (31). The survival rate of patients with IDO-1 expression was better than that of patients without it. IDO-1 expression of the UPS cell lines tended to react more strongly to IFN- $\gamma$ stimulation than that of PD-L1. The findings also suggest that IDO-1 may reflect the condition of anti-cancer immunity rather than PD-L1, and that immune reactions against tumor cells may work better in tumors with IDO-1 expression than in tumors without it. It was reported that tumor-infiltrating CD8-positive cells were predictive factor for anti-PD-1 immunotherapy (32). We considered that IDO-1 may be a predictive factor of the efficacy of anti-PD-1 therapy. It was also reported that tumors expressing IDO-1 may be a good target for anti-PD-1 therapy (12). Findings of that study may support our consideration that IDO-1 reflected the condition of anti-cancer immunity. In a previous study, it was reported that patients with IDO-1-positivity in stromal cells had better prognosis (33). In that study, the authors discussed that decreased tryptophan availability disturbs the proliferation of tumor cells to some extent. In the current study, macrophages and lymphocytes were stained by IDO-1 as well as tumor cells. In addition, IFN- $\gamma$ secreted by macrophages may stimulate themselves to express IDO-1. It was difficult to score the IDO-1 expression of stromal cells as described in a previous study as tumor and stromal cells were not clearly separated optically (33). In the current study as well, it was suggested that the exhaustion of tryptophan may interfere with tumor cell proliferation.

As in previous results from a meta-analysis (34), high expression of PD-L1 was related to an unfavorable prognosis in this study. This could be explained by tumor cells evading tumor immunity due to a high expression of PD-L1 potentially causing poor prognosis. By contrast, IDO-1 expression was related to a favorable prognosis, although IDO-1 may enable tumor cells to avoid the immune system. As mentioned above, IDO-1 exhibited close correlations with CD8 and CD3, thus the favorable effect of IDO-1 expression on prognosis may 

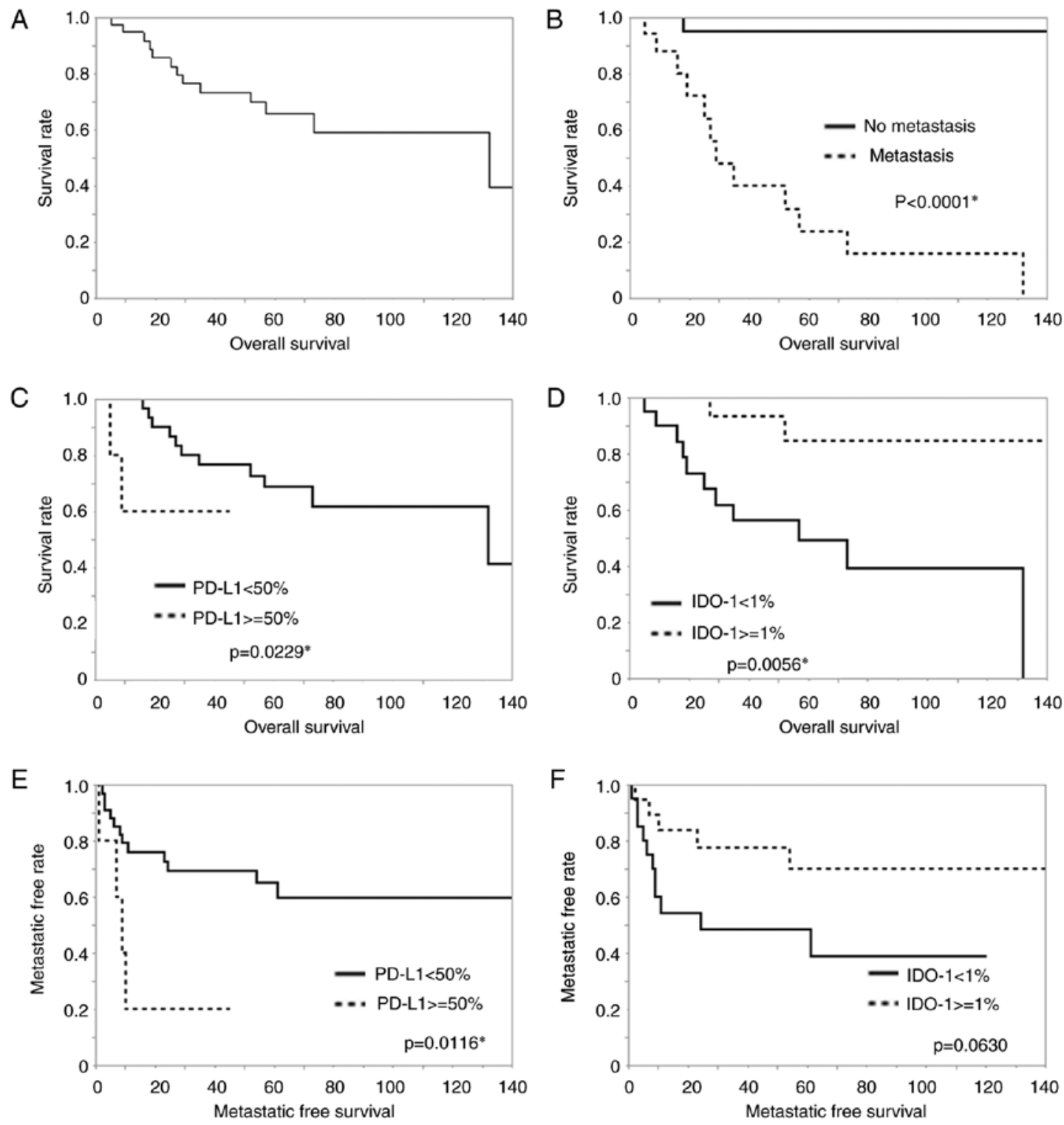

Figure 5. Kaplan-Meier curves of overall survival, analyzed by log-rank test (A-D). (A) Overall survival. (B) Metastasis was a factor associated with worse prognosis. (C) Patients with strong PD-L1 expression had worse prognosis. (D) Patients with at least focal IDO-1 expression had favorable prognosis. Other factors were not significantly related to overall survival. Kaplan-Meier curve of distant metastasis-free survival analyzed by log-rank test (E and F). (E) Patients with high PD-L1 expression had worse prognosis regarding metastasis. (F) Patients with focal IDO-1 expression ( $\geq 1 \%)$ tended to have favorable prognosis regarding metastasis. Other factors were not associated with metastasis. No factors were associated with local recurrence in this study.

reflect the promotion of the tumor-targeting immune system by TILs.

As for the clinicopathological features, overall survival and metastasis were closely related to each other as in a previous study (35). Specifically, the cases without metastasis tended to be less likely to result in tumor-related death. Therefore, it was suggested that wide resection before metastasis may be one of the most important therapeutic options and that a definitive diagnosis of UPS in the early therapeutic phase may be essential. In the present study, the existence of a focal myxoid matrix was not significantly associated with overall, metastasis-free, or recurrence-free survival.

Cases of UPS with tumor necrosis also expressed PD-L1 more often than those of UPS without it. This may depend on the TILs around the area of tumor necrosis. PD-L1 expression in these tumors may not necessarily reflect that tumor immunity is working. Therefore, anti-PD-1 therapy may not be useful for such tumors, although they expressed PD-L1.

In the present investigation, we found a small population of UPS cases with dMMR, which also expressed PD-L1. Regarding HLA class I, six tumors lost their expression and one of these expressed focal PD-L1. More studies on the effectiveness of anti-PD-1 therapy for tumors with the loss of HLA class I are needed.

As for the limitations of the current investigation, UPS is extremely rare, thus, the number of cases was small. There were only five UPS cases expressing PD-L1 ( $\geq 50 \%)$. As such, the possibility of bias in the results cannot be ruled out. The functional experiments on the usefulness of anti-PD1 or anti-PDL1 therapy to UPS cell lines were also missing. Our experiments were limited to the induction of INF- $\gamma$ to 
UPS cell lines and its effect on PDL-1 and IDO-1 expression. Our in vitro study showed only a preliminary result that IDO-1 expression induced by IFN- $\gamma$ stimulation in UPS cells tended to be stronger than PD-L1 expression. The results of double staining did not prove that TILs positive for CD8-, CD4-, and CD3-secreted IFN- $\gamma$ because of the data based on only immunochemical staining of the specimens. In addition, the outcome of anti-PD-1 therapy for patients with UPS was not included in the analysis. Therefore, the difference of effectiveness of anti-PD-1 therapy in UPS cases between UPS with and without IDO-1 expression remains uncertain. A clinical study with a larger number of UPS cases should be carried out.

In conclusion, findings of the present study showed that UPS tumor cells frequently expressed PD-L1 and IDO-1. It was also suggested that strong PD-L1 expression $(\geq 50 \%)$ requires factors other than TILs. Finally, PD-L1 expression $(\geq 50 \%)$ may be a poor prognostic factor and IDO-1 expression may be a better prognostic factor of for UPS patients.

\section{Acknowledgements}

We would like to thank all of the technical staff of the Department of Pathology, Kyushu University for their assistance. We also appreciate the technical assistance from The Research Support Center, Kyushu University Graduate School of Medical Sciences.

\section{Funding}

The present study was supported by the Japan Society for the Promotion of Science KAKENHI (19H03444).

\section{Availability of data and materials}

All data generated or analyzed during this study are included in this published article.

\section{Authors' contributions}

SI, YY, TI, KK HY and YO designed the study. SI, YY and MY collected the materials. SI, TI and YT performed the experiments. YM and YN collected the clinical information. SI wrote the manuscript. KK and YO reviewed and checked the manuscript. All authors read and approved the final manuscript.

\section{Ethics approval and consent to participate}

The present study was approved by the Kyushu University Committee of Bioethics (approval no. 29-429 and 29-625; 2017).

\section{Patient consent for publication}

Not applicable.

\section{Competing interests}

The authors declare that they have no competing interests.

\section{References}

1. The International Agency for Research on Cancer (IARC), Fletcher CDM, Chibon F and Mertens F: Pathology and genetics of tumours of soft tissue and bone (IARC WHO Classification of Tumours). IARC Press, Lyon, pp235-238, 2013.

2. Gronchi A, Palmerini E, Quagliuolo V, Broto JM, Pousa AL, Grignani G, Brunello A, Blay JY, Tendero O, Beveridge RD, et al: Neoadjuvant chemotherapy in high-risk soft tissue sarcomas: Final results of a randomized trial from Italian (ISG), Spanish (GEIS), French (FSG), and Polish (PSG) sarcoma groups. J Clin Oncol 1: 2178-2186, 2020.

3. Kim JH, Park HS, Heo SJ, Kim SK, Han JW, Shin KH, Kim SH, Hur H, Kim KS, Choi YD, et al: Differences in the efficacies of pazopanib and gemcitabine/docetaxel as second-line treatments for metastatic soft tissue sarcoma. Oncology 96: 59-69, 2019.

4. Nakamura T, Tsukushi S, Asanuma K, Katagiri H, Ikuta K, Nagano A, Kozawa E, Yamada S, Shido Y, Yamada K, et al: The clinical outcome of eribulin treatment in Japanese patients with advanced soft tissue sarcoma: A tokai musculoskeletal oncology consortium study. Clin Exp Metastasis 36: 343-350, 2019.

5. Schalper KA, Carvajal-Hausdorf D, McLaughlin J, Altan M, Velcheti V, Gaule P, Sanmamed MF, Chen L, Herbst RS and Rimm DL: Differential expression and significance of PD-L1, IDO-1, and B7-H4 in human lung cancer. Clin Cancer Res 15: 370-378, 2017.

6. Pardoll DM: The blockade of immune checkpoints in cancer immunotherapy. Nat Rev Cancer 22: 252-264, 2012.

7. Syn NL, Teng MW, Mok TS and Soo RA: De-Novo and acquired resistance to immune checkpoint targeting. Lancet Oncol 18: e731-e741, 2012.

8. Ready N, Hellmann MD, Awad MM, Otterson GA, Gutierrez M, Gainor JF, Borghaei H, Jolivet J, Horn L, Mates M, et al: First-Line nivolumab plus ipilimumab in advanced non-small-cell lung cancer (CheckMate 568): Outcomes by programmed death ligand 1 and tumor mutational burden as biomarkers. J Clin Oncol 20: 992-1000, 2019.

9. Tawbi HA, Burgess M, Bolejack V, Van Tine BA, Schuetze SM, Hu J, D'Angelo S, Attia S, Riedel RF, Priebat DA, et al: Pembrolizumab in advanced soft-tissue sarcoma and bone sarcoma (SARC028): A multicentre, two-cohort, single-arm, open-label, phase 2 trial. Lancet Oncol 18: 1493-1501, 2017

10. Marabelle A, Le DT, Ascierto PA, Di Giacomo AM, De Jesus-Acosta A, Delord JP, Geva R, Gottfried M, Penel N, Hansen AR, et al: Efficacy of pembrolizumab in patients with noncolorectal high microsatellite instability/mismatch repair-deficient cancer: Results from the phase II KEYNOTE-158 study. J Clin Oncol 1: 1-10, 2020.

11. Flores-Martin JF, Perea F, Exposito-Ruiz M, Carretero FJ, Rodriguez T, Villamediana M, Ruiz-Cabello F, Garrido F, Cózar-Olmo JM and Aptsiauri N: A combination of positive tumor HLA-I and negative PD-L1 expression provides an immune rejection mechanism in bladder cancer. Ann Surg Oncol 26: 2631-2639, 2019.

12. Johnson DB, Bordeaux J, Kim JY, Vaupel C, Rimm DL, Ho TH, Joseph RW, Daud AI, Conry RM, Gaughan EM, et al: Quantitative spatial profiling of PD-1/PD-L1 interaction and HLA-DR/IDO-1 predicts improved outcomes of anti-PD-1 therapies in metastatic melanoma. Clin Cancer Res 1: 5250-5260, 2018.

13. Munn DH and Mellor AL: Indoleamine 2,3 dioxygenase and metabolic control of immune responses. Trends Immunol 34: 137-143, 2013.

14. Prendergast GC, Smith C, Thomas S, Mandik-Nayak L, LauryKleintop L, Metz R and Muller AJ: Indoleamine 2,3-dioxygenase pathways of pathogenic inflammation and immune escape in cancer. Cancer Immunol Immunother 63: 721-735, 2014.

15. Long GV, Dummer R, Hamid O, Gajewski TF, Caglevic C, Dalle S, Arance A, Carlino MS, Grob JJ, Kim TM, et al: Epacadostat plus pembrolizumab versus placebo plus pembrolizumab in patients with unresectable or metastatic melanoma (ECHO-301/KEYNOTE-252): A phase 3, randomised, double-blind study. Lancet Oncol 20: 1083-1097, 2019.

16. Tumeh PC, Harview CL, Yearley JH, Shintaku IP, Taylor EJ, Robert L, Chmielowski B, Spasic M, Henry G, Ciobanu V, et al: PD-1 blockade induces responses by inhibiting adaptive immune resistance. Nature 27: 568-571, 2014.

17. Sznol M and Chen L: Antagonist antibodies to PD-1 and B7-H1 (PD-L1) in the treatment of advanced human cancer. Clin Cancer Res 19: 1021-1034, 2013. 
18. Yoshimoto M, Yamada Y, Ishihara S, Kohashi K, Toda Y, Ito Y, Yamamoto H, Furue M, Nakashima Y and Oda Y: Comparative study of myxofibrosarcoma with undifferentiated pleomorphic sarcoma: Histopathologic and clinicopathologic review. Am J Surg Pathol 44: 87-97, 2020.

19. Thway K, Flora R, Shah C, Olmos D and Fisher C: Diagnostic utility of p16, CDK4, and MDM2 as an immunohistochemical panel in distinguishing well-differentiated and dedifferentiated liposarcomas from other adipocytic tumors. Am J Surg Pathol 36: 462-469, 2012.

20. Edge SB, Byrd DR, Compton CC, Fritz AG, Greene FL and Trotti A (eds): AJCC Cancer Staging Manual. 7th edition. Springer, St. Louis, MO, 2010

21. Volaric A,GentzlerR,HallR,MehaffeyJH,StelowEB,BullockTN, Martin LW and Mills AM: Indoleamine-2,3-dioxygenase in non-small cell lung cancer: A targetable mechanism of immune resistance frequently coexpressed with PD-L1. Am J Surg Pathol 42: 1216-1223, 2018.

22. Herbst RS, Baas P, Kim DW, Felip E, Pérez-Gracia JL, Han JY, Molina J, Kim JH, Arvis CD, Ahn MJ, et al: Pembrolizumab versus docetaxel for previously treated, PD-L1-positive, advanced non-small-cell lung cancer (KEYNOTE-010): A randomised controlled trial. Lancet 9: 1540-1550, 2016.

23. Reck M, Rodriguez-Abreu D, Robinson AG, Hui R, Csőszi T, Fülöp A, Gottfried M, Peled N, Tafreshi A, Cuffe S, et al: Pembrolizumab versus chemotherapy for PD-L1-positive non-small-cell lung cancer. N Engl J Med 10: 1823-1833, 2016.

24. Seeber A, Klinglmair G, Fritz J, Steinkohl F, Zimmer KC, Aigner F, Horninger W, Gastl G, Zelger B, Brunner A and Pichler R: High IDO-1 expression in tumor endothelial cells is associated with response to immunotherapy in metastatic renal cell carcinoma. Cancer Sci 109: 1583-1591, 2018.

25. Perea F, Bernal M, Sánchez-Palencia A, Carretero J, Torres C, Bayarri C, Gómez-Morales M, Garrido F and Ruiz-Cabello F: The absence of HLA class I expression in non-small cell lung cancer correlates with the tumor tissue structure and the pattern of T cell infiltration. Int J Cancer 15: 888-899, 2017.

26. Nakano K, Yamamoto H, Fujiwara M, Koga Y, Tsuruta S, Ihara E, Oki E, Nakamura M, Ogawa Y and Oda Y: Clinicopathologic and molecular characteristics of synchronous colorectal carcinoma with mismatch repair deficiency. Am J Surg Pathol 42: 172-182, 2018.

27. Yoo SH, Keam B, Ock CY, Kim S, Han B, Kim JW, Lee KW, Jeon YK, Jung KC, Chung EJ, et al: Prognostic value of the association between MHC class I downregulation and PD-L1 upregulation in head and neck squamous cell carcinoma patients. Sci Rep 22: 7680, 2019.
28. Hakozaki M, Hojo H, Sato M, Tajino T, Yamada H, Kikuchi S and Abe M: Establishment and characterization of a new cell line, FPS-1, derived from human undifferentiated pleomorphic sarcoma, overexpressing epidermal growth factor receptor and cyclooxygenase-2. Anticancer Res 26: 3393-3401, 2006.

29. Nishio J, Iwasaki $H$, Nabeshima K, Ishiguro $M$, Isayama $T$ and Naito M: Establishment of a new human pleomorphic malignant fibrous histiocytoma cell line, FU-MFH-2: Molecular cytogenetic characterization by multicolor fluorescence in situ hybridization and comparative genomic hybridization. J Exp Clin Cancer Res 24: 153, 2010.

30. Boxberg M, Steiger K, Lenze U, Rechl H, von Eisenhart-Rothe R, Wörtler K, Weichert W, Langer R and Specht K: PD-L1 and PD-1 and characterization of tumor-infiltrating lymphocytes in high grade sarcomas of soft tissue-prognostic implications and rationale for immunotherapy. Oncoimmunology 20: e1389366, 2017.

31. Burr ML, Sparbier CE, Chan YC, Williamson JC, Woods K, Beavis PA, Lam EY,Henderson MA, Bell CC, Stolzenburg S, et al: CMTM6 maintains the expression of PD-L1 and regulates anti-tumour immunity. Nature 7: 101-105, 2017.

32. Sun R, Limkin EJ, Vakalopoulou M, Dercle L, Champiat S, Han SR, Verlingue L, Brandao D, Lancia A, Ammari S, et al: A radiomics approach to assess tumour-infiltrating $\mathrm{CD} 8$ cells and response to anti-PD-1 or anti-PD-L1 immunotherapy: An imaging biomarker, retrospective multicohort study. Lancet Oncol 19: 1180-1191, 2018.

33. Patil PA, Blakely AM, Lombardo KA, Machan JT, Miner TJ, Wang LJ, Marwaha AS and Matoso A: Expression of PD-L1, indoleamine 2,3-dioxygenase and the immune microenvironment in gastric adenocarcinoma. Histopathology 73: 124-136, 2018.

34. Zheng C, You W, Wan P, Jiang X, Chen J, Zheng Y, Li W, Tan J and Zhang S: Clinicopathological and prognostic significance of PD-L1 expression in sarcoma: A systematic review and meta-analysis. Medicine (Baltimore) 97: e11004, 2018.

35. Vasileios KA, Eward WC and Brigman BE: Surgical treatment and prognosis in patients with high-grade soft tissue malignant fibrous histiocytoma of the extremities. Arch Orthop Trauma Surg 132: 955-961, 2012.

36. Roland CL, May CD, Watson KL, Al Sannaa GA, Dineen SP, Feig R, Landers S, Ingram DR, Wang WL, Guadagnolo BA, et al: Analysis of clinical and molecular factors impacting oncologic outcomes in undifferentiated pleomorphic sarcoma. Ann Surg Oncol 23: 2220-2228, 2016. 